\title{
Unusual case of pulmonary valve atresia
}

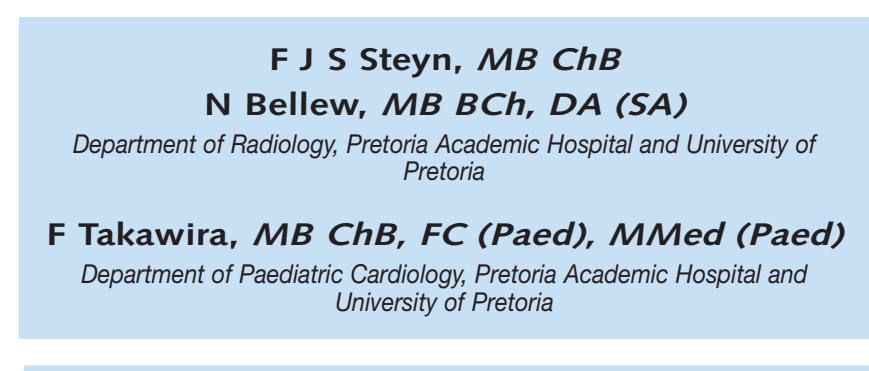

\section{Abstract}

We report on a 12-year-old boy with a rare form of pulmonary valve atresia with a ventricular septal defect (VSD) and anomalous origin of the left pulmonary artery arising from the aortic arch. He also has an absent right pulmonary artery, the right lung being supplied by small major aorto-pulmonary collaterals (MAPCAs). ${ }^{1} \mathrm{He}$ has decreased pulmonary blood flow to his right lung and increased pulmonary blood flow with irreversible pulmonary hypertension (PHT) on the left. Due to the late presentation, he was deemed inoperable due to the severe PHT of the left lung. ${ }^{2}$

\section{Introduction}

Pulmonary valve atresia with a VSD is an extreme form of tetralogy of Fallot (TOF). ${ }^{3}$ It usually presents in the neonatal period as a ductaldependent lesion and can be fatal without early surgical palliation. ${ }^{2}$ Anomalous origin of either pulmonary artery branch from the aorta has been reported. The association of pulmonary atresia (PA) with anomalous origin of the left pulmonary artery and an absent right pulmonary artery is, however, a very rare occurrence.-5

The best estimates of the relative frequency of PA-VSD are $2.5-3.4 \%$ of all congenital cardiac malformations. ${ }^{3,5-7}$ PA-VSD is slightly more prevalent in males than in females. ${ }^{3}$

\section{Case report}

We report on a 12-year-old boy who was referred to our cardiology service for evaluation due to his decreased effort tolerance, palpitations and coughing up of blood-stained sputum. Clinical examination revealed an appropriately grown boy with no dysmorphic features. Mild central cyanosis with oxygen saturation of $85 \%$ and digital clubbing were evident. His pulse rate was 90 beats/min. All his pulses were equally palpable but bounding in character. A wide pulse pressure with a blood pressure of $110 / 50 \mathrm{mmHg}$ was present. There was cardiomegaly with the apex beat in the 5th left intercostal space, lateral to the mid-clavicular line. A loud, single second heart sound was audible and no murmur was present. There were no signs of congestive cardiac failure.

Chest radiography (Fig. 1) revealed cardiomegaly (boot-shaped), with a cardiothoracic index (CTI) $>60 \%$, and an absent pulmonary bay. There was differential perfusion with increased pulmonary blood flow to the left lung relative to the right, and volume loss of the right lung, with

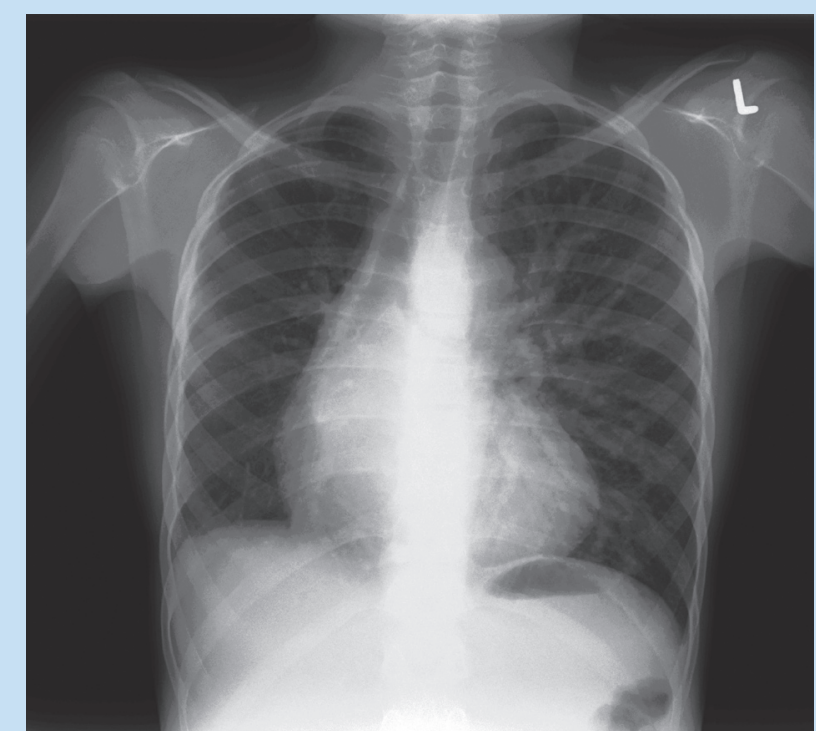

Fig. 1. Chest radiograph.

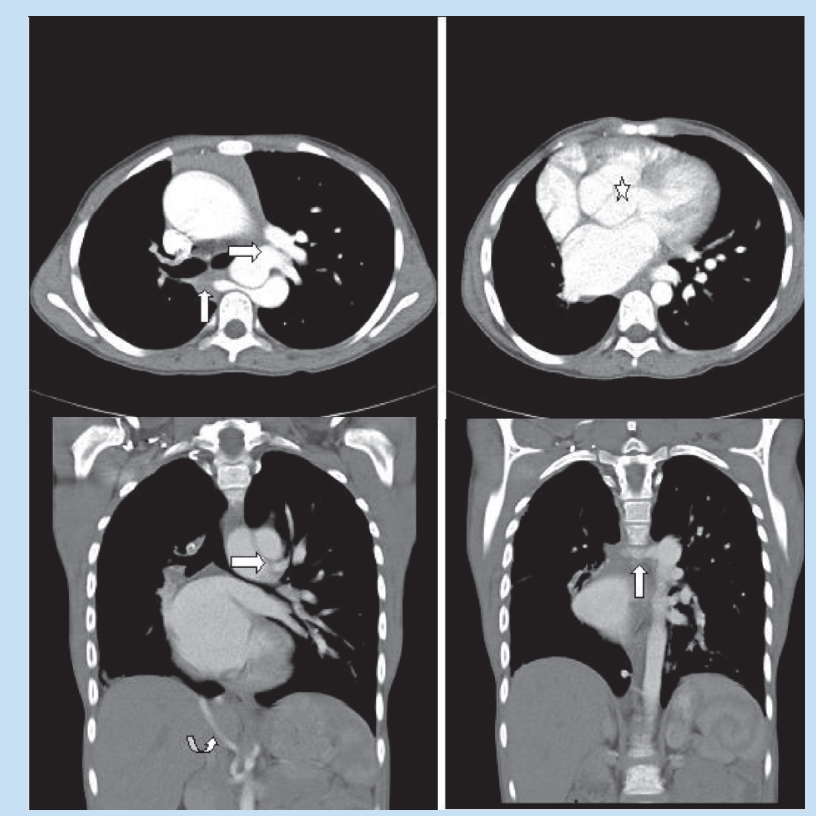

Fig. 2. CT angiogram demonstrates the left $(\Longrightarrow)$ as well as the hypoplastic right pulmonary artery (1) originating from the aortic arch. Note the overriding arterial trunk ( $\Sigma^{3}$ ) as well as the aberrant right pulmonary artery (u).

associated oligaemia and hyperinflation of the left lung with increased pulmonary vasculature. Electrocardiography showed left atrial enlargement and left ventricular hypertrophy.

Echocardiography demonstrated pulmonary valve atresia with a large VSD. No main pulmonary artery segment could be visualised. The left pulmonary artery (LPA) branch arose directly from the aortic arch. 
No right pulmonary artery (RPA) branch could be visualised, and there were indications of multiple collateral arteries supplying the lungs.

Conventional pulmonary angiography confirmed an LPA arising from the aortic arch, with no LPA stenosis and presumed to be at systemic aortic pressure, supplying the left lung. There was a blind-ending stump of the RPA arising from the descending aorta, giving rise to a few small MAPCAs supplying the right upper lobe. The right lower lung was perfused by an additional collateral from the abdominal aorta.

A pulmonary computed tomographic angiogram demonstrated a prominent overriding aortic outflow tract with a VSD and no pulmonary trunk. A large-calibre left pulmonary artery arose from the aortic arch. There was a hypoplastic right pulmonary artery arising from the proximal descending aorta, supplying the right upper lung zone. A hypoplastic right lung with a compensatory hyperinflated left lung was noted. An aberrant right pulmonary artery arising from the abdominal aorta at the origin of the superior mesenteric artery (SMA) supplying the right lower lung zone was also demonstrated.

\section{Discussion}

PA-VSD is a cyanotic congenital heart disease characterised by underdevelopment of the right ventricular (RV) outflow tract (i.e. subpulmonary infundibulum) with atresia of the pulmonary valve, a large VSD, and overriding of the aorta. In the past, this anomaly was termed pseudotruncus or truncus arteriosus type $4.1,5,8$

PA-VSD demonstrates a wide spectrum of severity, depending on the degree of pulmonary artery development. ${ }^{2}$ Pathologically, PA-VSD is frequently considered the most severe end of the spectrum of TOF, but controversy exists as to whether PA-VSD and TOF should be treated as two distinct entities. ${ }^{4-7}$ Unlike PA-VSD, patients with the standard type of TOF with pulmonary atresia have pulmonary arteries that are usually normal in size with normal peripheral pulmonary arborisation. In addition, systemic-to-pulmonary collateral vessels are not as well developed in patients with TOF with pulmonary atresia as they are in patients with PA-VSD. ${ }^{4,6}$

Our patient has a congenital cyanotic heart defect which is characterised by the rare combination of increased pulmonary blood flow to the left lung, with development of unilateral pulmonary hypertension, and decreased blood flow to the right lung, with under-perfusion of that lung. ${ }^{1,3,4}$ Because of his late presentation, he already had significant pulmonary hypertension; therefore, surgical palliation was no longer feasible or advisable. ${ }^{2}$

This patient presented with an overriding aortic arch, VSD, pulmonary valve atresia, absent main pulmonary artery (MPA), and an LPA and a hypoplastic RPA arising from the aorta. The left lung was hyperinflated with increased pulmonary vasculature, and the right lung was hypoplastic with aberrant supply from the abdominal aorta.

According to the classification of Collett and Edwards, this condition was classified as a type IV truncus arteriosus (pseudotruncus). It has been reclassified as pulmonary valve atresia with a VSD and MAPCAs by Van Praagh. ${ }^{5}$

The above-mentioned congenital heart disease represents less than $0.1 \%$ of all congenital heart diseases. ${ }^{3}$ Congenital obstruction of the right ventricular outflow tract leads to hypoplasia of the pulmonary arteries and persistence or hypertrophy of the primitive arterial connections to the lungs. Patients present with progressive dyspnoea and irreversible pulmonary hypertension.

\footnotetext{
1. Allanby KD, Brinton WD, Campbell M, Garnder F. Pulmonary atresia and the collateral circulation to the lungs. Guys Hosp Rep 1950; 99(2-3): 110-152.

2. Bharati S, Paul MH, Idriss FS, et al. The surgical anatomy of pulmonary atresia with ventricular septal defect pseudotruncus. J Thorac Cardiovasc Surg 1975; 69(5): 713-721.

3. Beauchesne LM, Warnes CA, Connolly HM, et al. Prevalence and clinical manifestations of 22q11.2 microdeletion in adults with selected conotruncal anomalies. J Am Coll Cardiol 2005; 45(4): 595-598.

4. Calder L, Van Praagh R, Van Praagh S, et al. Truncus arteriosus communis. Clinical, angiocardiographic, and pathologic findings in 100 patients. Am Heart J 1976; 92(1): 23-38.

5. Van Praagh R. Editorial: Classification of truncus arteriosus communis (TAC). Am Heart J 1976; 92(2) 129-132.

6. Mair DD, Julsrud PR. Diagnostic evaluation of pulmonary atresia and ventricular septal defect: Cardiac catheterization and angiography. Prog Pediatr Cardiol 1992; 1(1): 23-36

7. Somerville J. Management of pulmonary atresia. Br Heart J 1970; 32(5): 641-651.

8. d'Udekem Y, Alphonso N, Norgaard MA, et al. Pulmonary atresia with ventricular septal defects and majo aortopulmonary collateral arteries: unifocalization brings no long-term benefits. J Thorac Cardiovasc Sur 2005; 130(6): 1496-1502.
} 